INTERNATIONAL DESIGN CONFERENCE - DESIGN 2018

https://doi.org/10.21278/idc.2018.0158

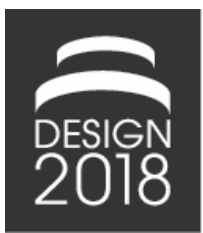

\title{
MATCHLINK - A MULTI-SENSORIAL GAME FOR PERSONS WITH DEMENTIA
}

\author{
C. E. L. Seah and M. T. K. B. Tan
}

\begin{abstract}
Sensory, cognitive and motor stimulation can enhance the wellbeing of people with dementia and their caregivers by reducing their susceptibility to developing behavioural or psychological symptoms. This project led to the development of a multi-sensorial game (MatchLink) for use by occupational therapists and caregivers to engage and stimulate people with dementia, with the intention to increase functional ability and prevent decline. Primary information was collected through an ethnographic observation at $\mathrm{Ng}$ Teng Fong Hospital with the guidance of an occupational therapist.
\end{abstract}

Keywords: dementia, multisensory product experience, cognitive stimulation, social innovation, product design

\section{Introduction}

Dementia refers to a 'syndrome of acquired loss of cognitive function, behavioural changes and loss of social function' (Jones, 2000). On a global level, it is one of the biggest global public health challenge with an estimated number of 44 million people affected by dementia worldwide in 2014 (Alzheimer's Disease International, 2014). The number is expected to double by 2030 and more than triple by the year 2050 (Alzheimer's Disease International, 2014). The cost of the global epidemic is estimated in 2010 to be US \$604 billion and possibly more (Alzheimer's Disease International, 2014). This paper investigates non-pharmacological interventions to cognitively and sensorially stimulate the senses of persons with dementia, as well as the creation of a game that combines the benefits from cognitive and sensory games. It also introduces an approach which allows caregivers and occupational therapists to access the player's abilities over time.

\section{Background}

The more common types of Dementia include Alzheimer's Disease, Vascular Dementia, Dementia with Lewy bodies and Frontotemporal dementia with Alzheimer's Dementia being the most common, accounting for $50-60 \%$ of dementias (Jones, 2000).

Presence of Dementia can be identified from these symptoms (Jones, 2000)

- Memory loss, mostly of recent events

- Decreased ability with learning and retaining new information

- Decreased ability to perform complex tasks

- Difficulty of reasoning capabilities, spatial and visuo-perceptual ability

- Language deficits

- Significant changes in behavior 
Persons with Dementia are found to have reduced social interaction and experience loneliness (Alzheimer's Society, 2013). They are susceptible to develop behavioural or psychological symptoms such as agitation, aggression, delusions, depression (Burns et al., 2002). Such experiences can have adverse effects on their well-being and increase the risk of deterioration (Burns et al., 2002).

\subsection{Treatments available}

\subsubsection{Antidementia drugs}

There are many compounds available targeting symptoms of Alzheimer's disease and other dementia with many being developed for Alzheimer's Disease rather than for dementia as a whole (Jones, 2000). Pharmacological strategy and treatment 'acting directly on muscarinic and nicotinic receptors or involving other neurotransmitters and growth factors may lead to even more effective therapies' (Jones, 2000). Non-drug interventions should be considered before starting drug treatment because of the problems that drug-based interventions bring (Jones, 2000).

\subsubsection{Non-pharmacological interventions}

Non-pharmacological interventions are used to alleviate behavioural and psychological symptoms of dementia. Core symptoms include cognitive, functional and social decline whereas associate symptoms encompasses agitation, aggression, depression, psychosis, repetitive vocalization, sleep disturbance and other non-specific behavior disturbances (SIGN, 2006). Intervention refers to behavior management, caregiver intervention programmes, cognitive stimulation, environmental design, multisensory stimulation and combined therapies, physical activities, reality orientation therapy, recreational actitivies, simulated presence and validation therapy (SIGN, 2006). For the purpose of this paper and product, focus will be on cognitive and multi-sensory stimulation.

\subsubsection{Cognitive stimulation and recreational activity}

According to SIGN, formal cognitive stimulation has shown to produce a positive results, people with dementia show improved memory of specific pieces of information but was not able to show benefits regarding memory function. Cognitive stimulation training's benefit however lies in minimal training of education and training of the caregiver as well as no risk to the person with dementia (SIGN, 2006). Some examples of cognitive stimulation include activities that provoke memory, encourages problem solving and conversational fluency (SIGN, 2006).

See Shape encourages users to find geometric blocks, following instructions to form shapes. It utilizes visual-spatial, construction and problem-solving skills. To play See Me, users identify pictures printed on each card and arrange them according to categories through association, training their memory skills.

\subsubsection{Social participation}

Social participation provides a wide range of health benefits for older adults, resulting in decreased risk of dementia and Alzheimer's Disease (Paillard-Borg et al., 2004) decreased risk of cognitive decline, depression and generalized anxiety disorders (Golden et al., 2009).

\subsubsection{Sensory stimulation}

Continue social interaction and cognitive stimulation can help promote the well-being of persons with Dementia (Alzheimer's Association, n.d.). Apart from the use of pharmacological approaches, Sensory Stimulation Therapy (SST) has provided another means to manage and care for PWD (Burns et al., 2002). Sensory stimulation therapy is used to arouse the senses - sight, smell, hearing, taste and touch. Sensory stimulation activities are able to improve on functional ability and the quality of life for PWDs as well as early and mid-stage probable Alzheimer's Disease (AD) (Witucki and Twibell, 1997). Sensory Stimulation presented with social interaction resulted in a decrease in psychological discomfort level for the elderly in the late stages of Alzheimer's Disease (Schmid, 2009). PWDs who undergo multi-sensorial stimuli showed evidence of rest and relaxation, better sleep patterns, lesser agitation and 
the reduced use of medication for pain (Kim, 2008). Patients can however be easily over stimulated and are believed to have a decreased sensory threshold. Balance is essential in terms of the type and intensity of stimulation as both deprivation and overstimulation may lead to behavioural or psychological symptoms over time (Milev et al., 2008).

Multi-sensory stimulation utilizes a variety of equipment like lighting effects, music, tactility and fragrances to create a multi-sensory effect. There are some benefits from using multisensory stimulation to relieve core symptoms for people with dementia, reducing confusion and speaking more spontaneously in normal length sentences (SIGN, 2006).

\subsubsection{Sensory games in the market}

Sensory blankets are patches of various tactile materials like zips, keychains, ribbons, button and bells stitched together, used to provide different sensations of textures and sounds. Sensory pouches are filled with everyday object that provide different textures for the elderly to identify and touch. Most sensory games use everyday objects, facilitating the use of touch and memory recall. It however limits the engagement to visual, precision, eye-hand coordination and recall skills.

\subsubsection{Scent guessing}

Games utilizing aroma are also ways to provide olfactory stimulation. These games uses aromatherapy through essential oils like peppermint, lavender, rosemary and other scents from fruits, flora and fauna. Games using scented therapeutic play dough like Jiak Ba Buay are fun, tactile and allows users to get creative, and create shapes and designs. They however can only be used once to provide for sanitary needs in the hospital environment where games have to be sanitized after each use. There is also a risk of elderly users unknowingly consuming the dough.

Our sense of touch influences our understanding of the environment around us (Song, 2010). Experiences of touch may influence what our mind experiences and helps us to make sense of the environment we live in (Song, 2010). It is helpful to utilise tactile elements to evoke memories and emotions together with social interaction to stimulate PWD's and reduce agitation (Schmid, 2009). According to a study conducted by neuroscientists of the Charité, the results show that a quantitative tactile memory representation exists in the frontal lobes of the human brain (Spitzer, 2011). The representation of the memory can be controlled consciously and is dependent on the individual's current memories (Spitzer, 2011).

\section{Methodology}

To facilitate design development, a week long participant observation was conducted at $\mathrm{Ng}$ Teng Fong General Hospital to observe, learn and gain insight to the activities and tools used by the occupational therapist for patients with dementia. Occupational therapists help patients to 'develop, improve, sustain or restore independence to any person who has an injury, illness, disability or psychological dysfunction' (Occupational Therapy, n.d.). Activities that the occupational therapists observed include observing patients performing cognitive and motor tasks, asking questions and reviewing their medical records, evaluating their condition and needs before developing a treatment plan. They also educate a patient's family on caregiving and identify improvements and potential fall hazards to meet patients' needs. Working with the elderly, occupational therapists help them to lead more active and independent lives.

The occupational therapist attached for this project has had more than 12 years of experience caring mainly for dementia patients. Patients were hospitalized for different diseases, not just specifically dementia and alzhiemer's disease but because of accompanying illnesses and injuries from falls. There was also a need for occupational therapists and nurses caring for patients to speak various languages ranging from English, Mandarin, Hindi, Malay and dialects like Hokkien, Teochew, Cantonese, Hakka and Hainanese because of the diverse backgrounds patients come from.

The rehabilitation process included activities carried out in the life hub, gym and activity room where patients are rehabilitated to improve their current condition through activities. In the gym, patients perform activities like climbing steps, lifting items and using a wide range of actions that are commonly used in their home environment to perform daily living activities. In the life hub, patients cook, clean 
and socialize, also performing activities crucial to daily life like hand cycling, and folding and putting on clothes or reaching out to hang clothes on poles. In the activity room, patients mainly engage in games with occupational therapists and nurses. These games stimulate patients cognitively and are used for occupational therapists to assess patients' abilities. There was great emphasis on sanitary requirements as each item was cleaned after each use. Portability and easy storage was necessary to facilitate game usage throughout different areas.

Current games used by the occupational therapists to engage and stimulate cognitive skills and motor function for patients with dementia include puzzles, bead sequencing, tangrams, animal jigsaws, Jenga and cone stacking. Most of the games relied on visual skills, precision, eye-hand coordination for matching colours, shapes, solving puzzles, which the occupational therapist could customize based on the patient. There was a lack of emphasis on tactile stimulation. Products that incorporate the use of dementia patient's senses can be incorporated into current occupational therapist treatments, expanding the range of tools that occupational therapists can utilize with the emphasis of tactile stimulation.

\section{Objectives}

This project aims to explore and develop a multi- sensorial game for use by occupational therapists and caregivers to engage PWD's to increase functional ability and promote cognitive stimulation. The objectives include incorporating sensory, visual, memory, precision, co-ordination, orientational and creative skills to stimulate cognitive processing and motor skills, through tactile and visual means. Many other sensory games in the market focus on the tactility or olfactory aspect without incorporating the various skills which can further cognitively stimulate people with dementia.

To ensure effectiveness of the game, a scorecard will be included in each game set for caregivers to assess abilities of players. This scorecard will also be used in Ng Teng Fong Hospital and data will be collected pertaining to whether long term usage of the game leads to better scores.

\section{Proposed idea}

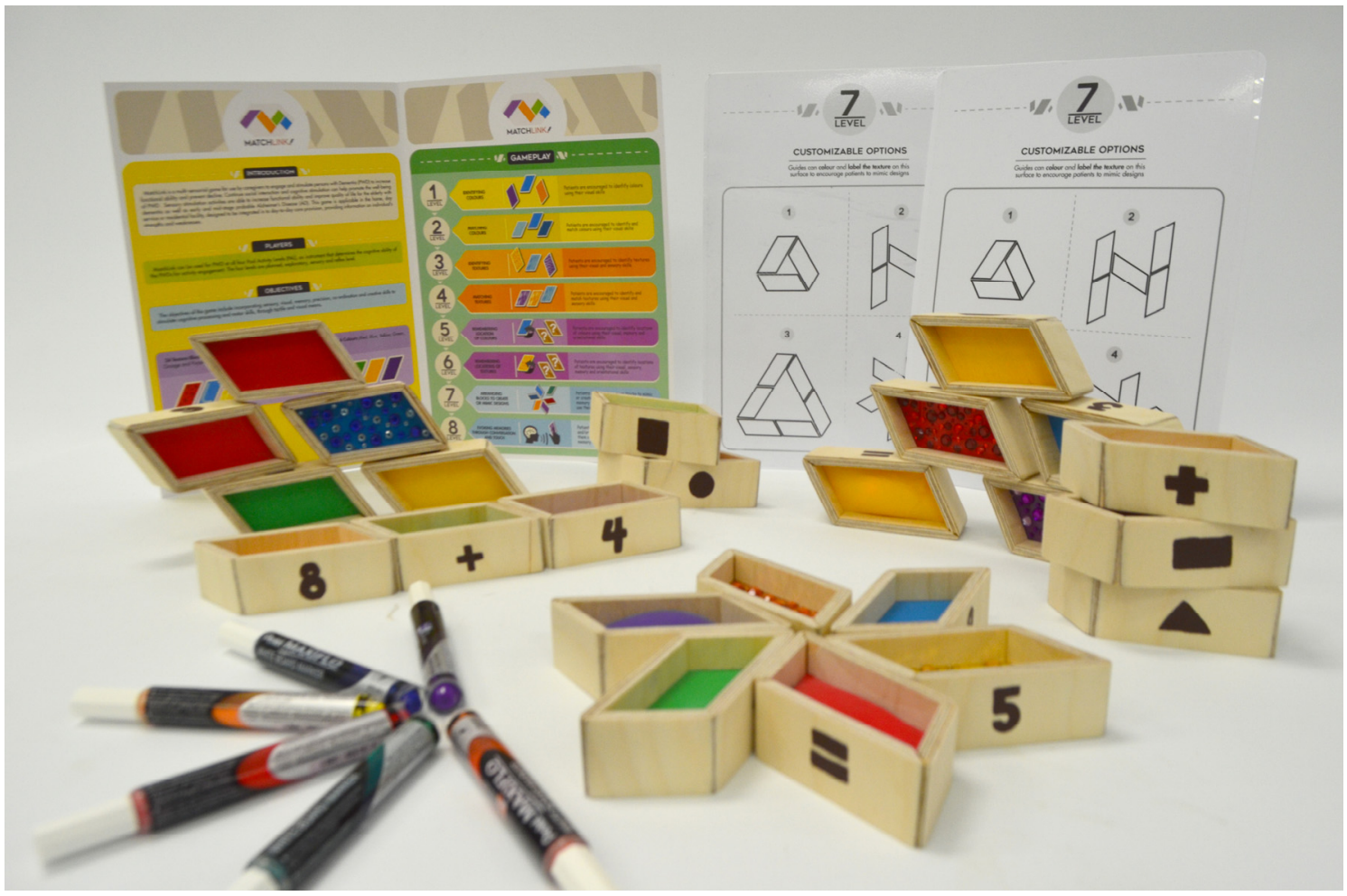

Figure 1. MatchLink game set components (Seah, 2017) 
I explored and created a multi-sensory game, MatchLink, with the emphasis on tactile stimulation that can help provide sensory and cognitive stimulation for people with dementia for use by occupational therapists. The game follows the needs of patients at all four Pool Activity Levels, planned, exploratory, sensory and reflex levels. MatchLink can be used for patients at all four Pool Activity Levels (PAL), an instrument that determines the cognitive ability of the people with dementia for activity engagement (Wenborn et al., 2008). The four levels are planned, exploratory, sensory and reflex level (Wenborn et al., 2008).

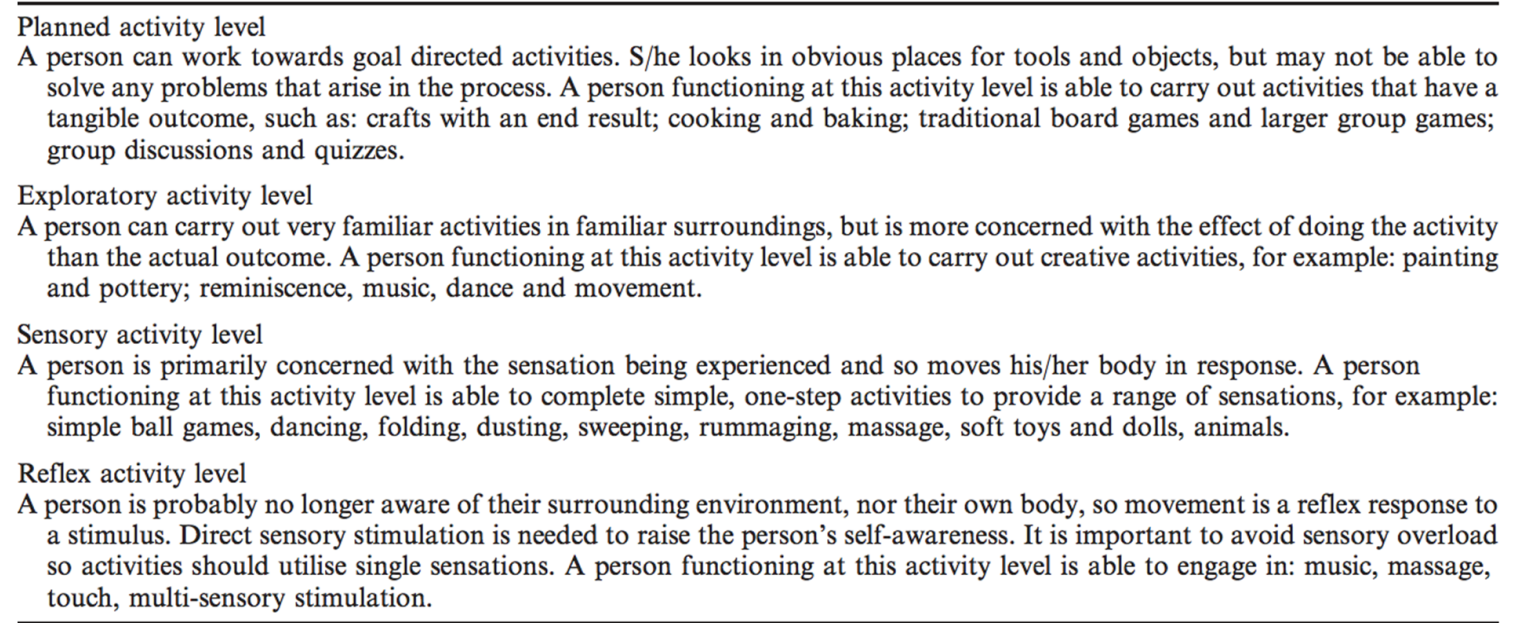

\section{Figure 2. Pool Activity Level (Wenborn et al., 2008)}

MatchLink has different levels of cognitive and sensory skills, which can be applied by occupational therapists or caregivers on people with varying intensity of dementia. The game has eight activities: identifying colours, matching colours, identifying textures, matching textures, remembering locations of colours, textures and arranging blocks to create designs. It will also be used as a means to evoke memories and emotions through interaction with players and touching varying textures they are familiar with.

The product has to be sanitized after each patient's use in the hospital setting. As such, materials such as acrylic, wood, textured surfaces and rubber will be used to meet the tactile and sanitary requirements. It can be flat packed and stored easily for easier portability. This game is applicable in the home, day service or residential facility, designed to be integrated in to day- to-day care provision, providing information on individual's strengths and weaknesses. It is meant be used flexibly in a one-on-one setting or with a group of players, amongst the elderly and their caretakers. Instruction manual for the game can be found in Appendices.

Smooth, rough, hard and soft textures are used and each texture has six different colours each, red, blue, yellow, green, orange and purple which are primary and secondary colours the elderly may be more familiar with.

\subsection{Game levels}

\section{Level 1: Identifying colours}

Caregiver places all 24 textured and coloured blocks on the table surface and ask the player to identify the chosen colour. Patients are encouraged to identify colours using visual skills. Number of blocks on the surface can be reduced if it is too stimulating for player. Caregivers may also pick out colours for players to identify individually.

\section{Level 2: Matching colours}

Caregiver places all 24 textured and coloured blocks on the table surface and picks out a colour for players to identify and then pick out the same colours from the table. Patients are encouraged to match colours using their visual, precision and co-ordination skills. Number of blocks on the surface can be reduced if it is too stimulating for player. 


\section{Level 3: Identifying textures}

Caregiver places all 24 textured and coloured blocks on the table surface and ask the player to identify the chosen texture. Patients are encouraged to identify textures using their visual and sensory skills. Caregivers can also pick out textures for players to identify individually. Number of blocks on the surface can be reduced if it is too stimulating for player.

\section{Level 4: Matching textures}

Caregiver places all 24 textured and coloured blocks on the table surface and picks out a texture for players to identify and then pick out the same textures from the table. Patients are encouraged to match textures using their visual, sensory, precision and co-ordination skills. Number of blocks on the surface can be reduced if it is too stimulating for player.

\section{Level 5: Remembering locations of colours}

From all textured and colour blocks, caregiver chooses a specific colour and places it among one to four blocks of a different colour depending on the player's capability. Caregiver then flips the set of blocks face down and encourages the player to identify and remember locations of colours using visual, memory and orientational skills.

\section{Level 6: Remembering location of textures}

From all textured and colour blocks, caregiver chooses a specific texture and places it among one to four blocks of a different texture depending on the player's capability. Caregiver then flips the set of blocks face down and encourages the player to identify and remember locations of textures using visual, sensory, memory and orientational skills.

\section{Level 7: Arranging blocks}

Caregiver uses whiteboard markers to colour one of the four shapes on the whiteboard, labeling each coloured block with a texture as well. Caregiver then lays all textured and coloured blocks for players to pick and match shapes to the given instructions. Players are encouraged to use blocks to mimic designs using visual, memory, sensory and comprehension skills. Should the difficulty level be too high, caregivers can remove the sensory component and focus solely on the colours before advancing further.

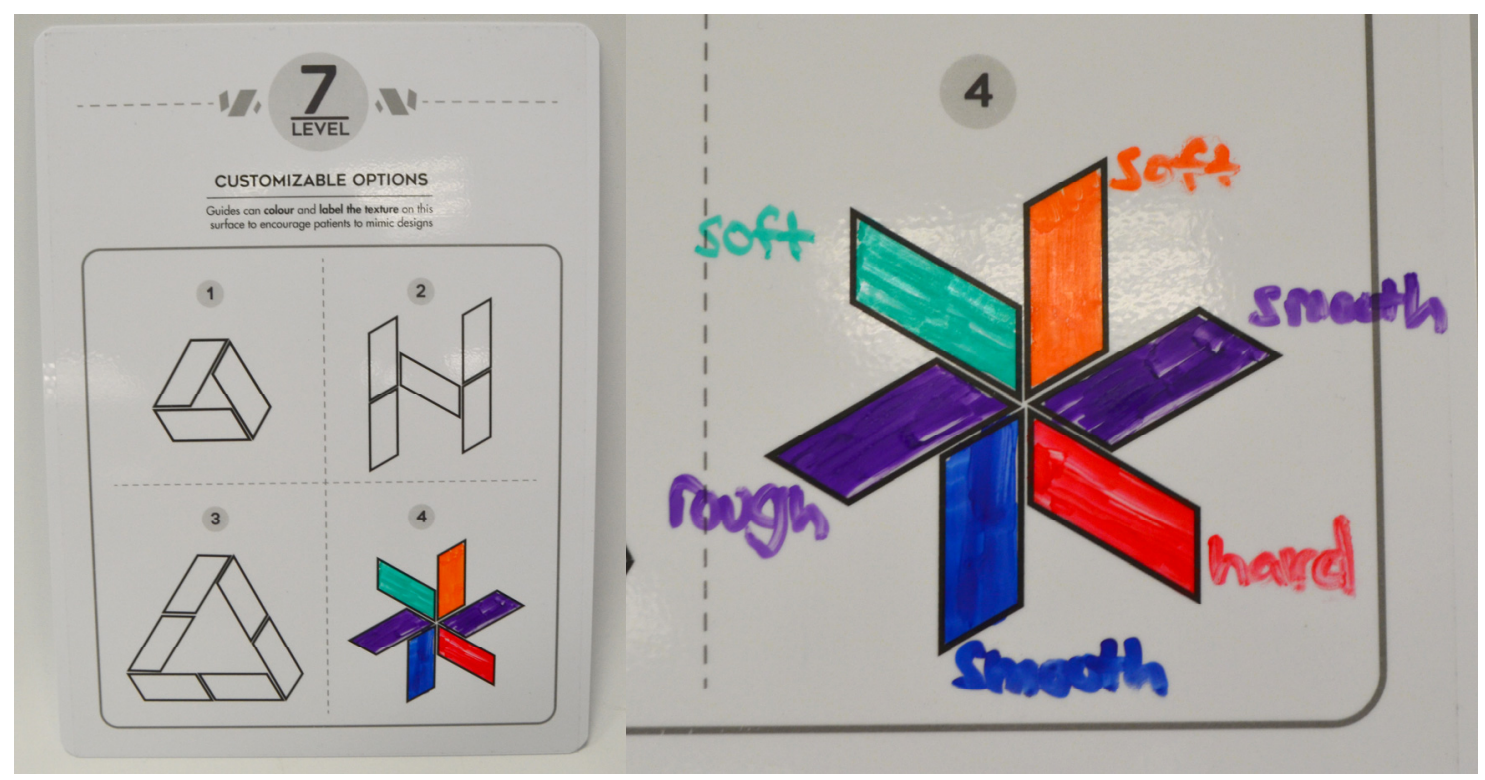

Figure 3. Texture and colours whiteboard with defined format (Seah, 2017) 
Players can also be given the choice get creative and to draw out shapes on the back of the whiteboard before colouring and labeling the blocks and forming them using the actual blocks. They can also form 3D shapes with blocks by stacking them. Players are encouraged to use blocks to create designs using visual, memory, sensory, comprehension and creative skills.

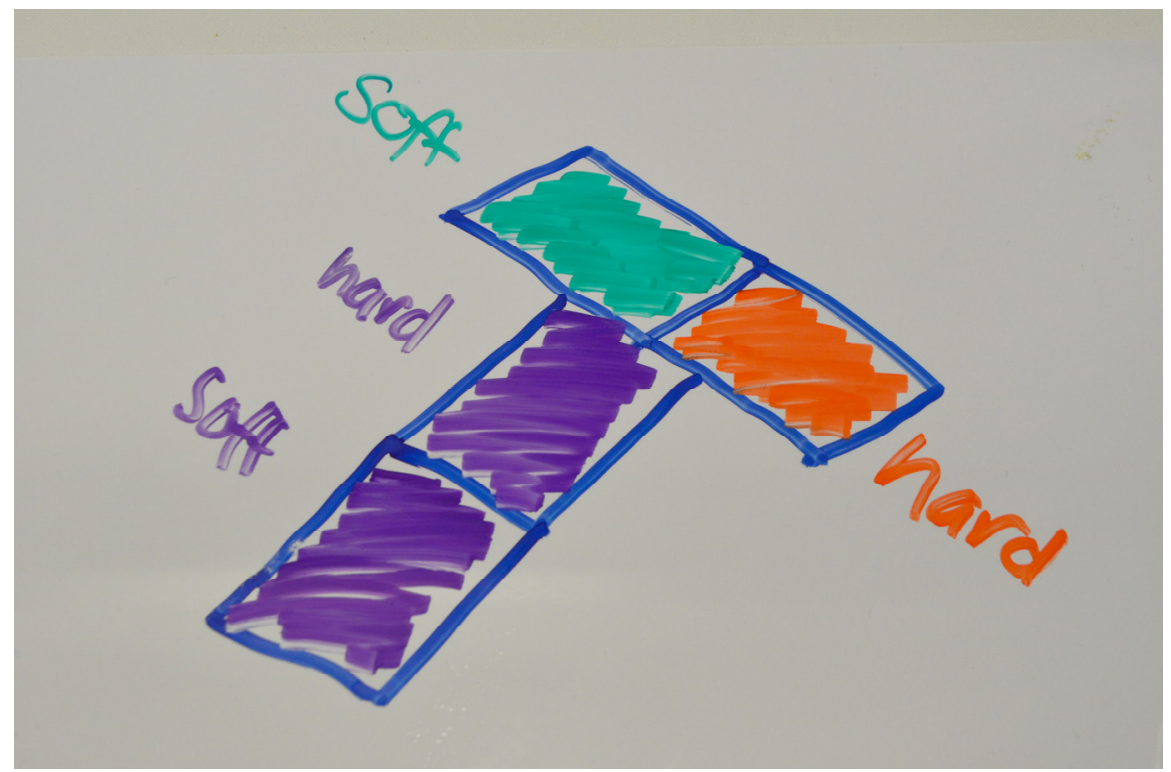

Figure 4. Texture and colours whiteboard (Seah, 2017)

Level 8: Evoking memories through conversation and touch

Players get to pick a texture and colour they are familiar with or wish to talk about. Caregivers may choose the texture and colour to guide the player as well. Players are encouraged to use visual, memory and sensory skills.

\subsection{Pool Activity Level and versatility}

Individuals at the planned stage can utilise game levels 1 to 8 . Exploratory stage individuals can engage with levels 1 to 6 and 8. Individuals at the sensory stage can interact with the game from levels 1 to 4 and 8 and reflex stage individuals will be able to benefit from levels 1 to 3 and 8 . Game difficulty levels can also be expanded or decreased from two up to five blocks in each level to suit player's capabilities.

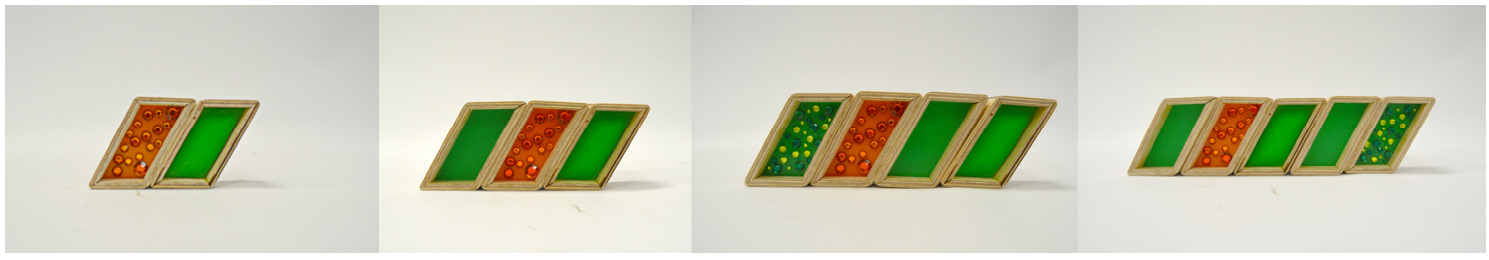

Figure 5. Range of expandable difficulty level (Seah, 2017)

\subsection{Score cards for design survey}

How players will be assessed will be based on this scorecard where points are given based on the number of blocks identified and the intensity of the given set of blocks. Player's capabilities will be assessed based on the increase in number of points from each game session. Having the scorecard makes it easy for caregivers to keep track of player's improvement and the skills they are able to use. 


\section{Points \\ System}

Level 1

\begin{tabular}{|c|c|c|c|c|c|c|}
\hline Colours Recognised & 1 & 2 & 3 & 4 & 5 & 6 \\
\hline Total Points & \multicolumn{10}{|l|}{} \\
\hline
\end{tabular}

Level 2

\begin{tabular}{|c|c|}
\hline Secs of Colours Matched & Points \\
\hline 1 & 3 \\
\hline 2 & 6 \\
\hline 3 & 9 \\
\hline 4 & 12 \\
\hline 5 & 15 \\
\hline 6 & 18 \\
\hline Total Points & \\
\hline
\end{tabular}

Level 4

\begin{tabular}{|c|c|}
\hline Secs of Textures Marched & Points \\
\hline 1 & 5 \\
\hline 2 & 10 \\
\hline 3 & 15 \\
\hline 4 & 20 \\
\hline Total Points & \\
\hline
\end{tabular}

Level 3

\begin{tabular}{|c|l|l|l|l|}
\hline Textures Recognised & 1 & 2 & 3 & 4 \\
\hline Total Points & \multicolumn{5}{|c|}{} \\
\hline
\end{tabular}

Level 5

\begin{tabular}{|c|c|c|c|c|}
\hline & 3 Blocks & 4 Blocks & 5 Blocks & 6 Blocks \\
\hline 2 Colours & 6 & 8 & 10 & 12 \\
\hline 3 Colours & 9 & 12 & 15 & 18 \\
\hline 4 Colours & 12 & 16 & 20 & 24 \\
\hline Total Points & \multicolumn{4}{|l}{} \\
\hline
\end{tabular}

Level 6

\begin{tabular}{|c|c|c|c|c|}
\hline & 3 Blocks & 4 Blocks & 5 Blocks & 6 Blocks \\
\hline 2 Textures & 6 & 8 & 10 & 12 \\
\hline 3 Textures & 9 & 12 & 15 & 18 \\
\hline 4 Textures & 12 & 16 & 20 & 24 \\
\hline Total Points & \multicolumn{5}{|l}{} \\
\hline
\end{tabular}

Level 7

\begin{tabular}{|c|c|}
\hline & Points \\
\hline Coloured Blocks & 5 \\
\hline Labelled Textures & 5 \\
\hline Correct Arrangement & 20 \\
\hline Total Points & \\
\hline
\end{tabular}

Level 8

\begin{tabular}{|c|c|}
\hline & Points \\
\hline Shallow Detail & 5 \\
\hline Moderate Detail & 10 \\
\hline In Depch Detail & 15 \\
\hline Total Points & \\
\hline
\end{tabular}

Figure 6. Score card system (Seah, 2017) 


\section{Level 1: Identifying colours}

Points are awarded based on the number if colours recognised with each block representing one point.

\section{Level 2: Matching colours}

Points are awarded based on the number of colour set matched. Caregiver identifies and takes out one colour leaving three other of the same colour to be picked out. With each set of colours matched, points are accumulated.

\section{Level 3: Identifying textures}

Points are awarded based on the number of textures recognised with each block representing one point.

\section{Level 4: Matching textures}

Points are awarded based on the number of texture set matched. Caregiver identifies and takes out one texture leaving five other of the same texture to be picked out. With each set of textures matched, points are accumulated.

\section{Level 5: Remembering locations of colours}

Caregivers arrange 3 to 6 blocks comprising of 2 to 4 different colours and collate the respective points when players achieve the objective.

\section{Level 6: Remembering location of textures}

Caregivers arrange 3 to 6 blocks comprising of 2 to 4 different textures and collate the respective points when players achieve the objective.

\section{Level 7: Arranging blocks}

5 points are allocated for colours shaded on the whiteboard, 5 more for textures labelled and 20 points for correctly mimicking the given shape on the whiteboard. 20 bonus points will be given should player draw out the shapes on their own on the back of the whiteboard.

\section{Level 8: Evoking memories through conversation and touch}

Players are assessed based on conversation with caregiver, 5 points are given for shallow memory recall and explanation. 10 points are given for a moderate conversation and 15 points for an in-depth conversation.

\section{Reflection}

The product was presented to the hospital for further discussion, refinement and testing. It has been used in the hospital by occupational therapists for the past 8 months and future iterations are being created based on patients' and occupational therapists' and nurses' feedback. Changes made were based on economical costs using printed textures to achieve a similar effect. Some of the challenges faced include deciding on block forms, textures to include and materials to use to extend the lifespan of the product. More research and testing can be done with regard to the ergonomics of each piece and the textural elements that the elderly interact with. Further focus can be on the stimulation different textures provide for the elderly and different levels can be modified to include more complex tasks or incorporate different types of textures.

\section{Conclusion}

The exploration and creation of the multi-sensorial game provides caregivers with an additional mean to engage and stimulate persons with dementia preventing decline and increasing ability with a greater emphasis on tactile stimulation. The incorporation of visual, sensory, precision, co-ordination, orientational, memory, comprehension and creative skills can potentially greater stimulate persons with Dementia cognitively and sensorially. MatchLink also provides caregivers with a tool to engage players and at the same time be able to have a gauge as to how the player is improving over time and what kinds of skills they can employ. 


\section{Acknowledgement}

This project would not have been possible without the support and guidance of Assistant Professor Michael Thaddeus Tan Koon Boon, from School of Art, Design and Media, Nanyang Technological University. I also wish to thank Too San Muan, a senior Occupational Therapist and Tan Hwee Ping, a Senior Assistant Director, at Ng Teng Fong Hospital for their constant support, patience, generosity and kindness throughout the entire project. I wish to acknowledge the funding support for this project from Nanyang Technological University under the Undergraduate Research Experience on Campus (URECA) programme.

\section{References}

Alzheimer's Association (n.d.), Prevention and Risk of Alzheimer's and Dementia. [online] Alzheimer's Association. Available at: http://www.alz.org/research/science/alzheimers_prevention_and_risk.asp (accessed 01.08.2016).

Alzheimer's Disease International (2014), World Alzheimer Report 2014: Dementia and Risk Reduction, Alzheimer's Disease International (ADI), London.

Alzheimer's Society (2013), Majority of people living alone with dementia feel lonely. [online] Alzheimer's Society. Available at: https://www.alzheimers.org.uk/dementia2013 (accessed 01.08.2016).

Burns, A., Byrne, J., Ballard, C. and Holmes, C. (2002), "Sensory stimulation in dementia: An effective option for managing behavioural problems", British Medical Journal, Vol. 325 No. 7376, pp. 1312-1313.

Golden, J., Ronan, C.M. and Lawlor, B.A. (2009), "Underlying dimensions and association with psychological and physical health", Psychology, Health \& Medicine, Vol. 14 No. 3, pp. 280-290. https://doi.org/10.1080/13548500902730135

Jones, R.W. (2000), Drug Treatment in Dementia, Blackwell Science Ltd, Oxford. https://doi.org/ $10.1002 / 9780470698747$

Kim, H.S. (2003), Applied Multi-Sensory Stimulation (AMSS) approach for the Dementia and Alzheimer's disease population: case study, $\mathrm{PhD}$ thesis, Union Institute and University.

Milev, R., Kellar, T., McLean, M., Mileva, V., Luthra, V. et al. (2008), "Multisensory Stimulation for Elderly With Dementia: A 24-Week Single-Blind Randomized Controlled Pilot Study", American Journal of Alzheimer's Disease \& Other Dementias, Vol. 23 No. 4, pp. 372-376. https://doi.org/10.1177/1533317508316681

Occupational Therapy (n.d.), A Definition of Occupational Therapy. [online] Department of Occupational Therapy, NYU Steinhardt. Available at: https://steinhardt.nyu.edu/ot/definition/

Paillard-Borg, S., Winblad, B. and Fratiglioni, L. (2004), "An active and socially integrated lifestyle in late life might protect against dementia", The Lancet Neurology, Vol. 3 No. 6, pp. 343-353. https://doi.org/10.1016/S1474-4422(04)00767-7

Schmid, J. (2009), Sensory Stimulation for Alzheimer's. [online] Best Alzheimer's Products. Available at: http://www.best-alzheimers- products.com/sensory-stimulation-for-alzheimers.html (accessed 15.09.2016).

SIGN (2006), Management of Patients with Dementia, Scottish Intercollegiate Guidelines Network, Edinburgh.

Song, S. (2010), How Things You Touch Influence the Way You Think. [online] Time. Available at: http:/healthland.time.com/2010/06/24/study-how-things-you-touch-influence-the-way-you-think/ (accessed 09.03.2017).

Spitzer, B. (2011), How a person remembers a touch. [online] Universitatzmedizin. Available at: https://www.charite.de/en/service/press_reports/artikel/detail/wie_sich_der_mensch_an_eine_beruehrung_eri nnert/ (accessed 09.03.2017).

Wenborn, J., Challis, D., Pool, J., Burgess, J., Elliott, N. and Orrell, M. (2008), "Assessing the validity and reliability of the Pool Activity Level (PAL) Checklist for use with older people with dementia", Aging \& Mental Health, Vol. 12 No. 2, pp. 202-211. https://doi.org/10.1080/13607860801984375

Witucki, J.M. and Twibell, R.S. (1997), "The effect of sensory stimulation activities on the psychological well being of patients with advanced Alzheimer's disease", American Journal of Alzheimer's Disease \& Other Dementias, Vol. 12 No. 1, pp. 10-15. https://doi.org/10.1177/153331759701200103

\section{Appendix}

The user manual to facilitate participation amongst the elderly. Level 7 would come in the form of a whiteboard to allow for customization of textures and colours. 

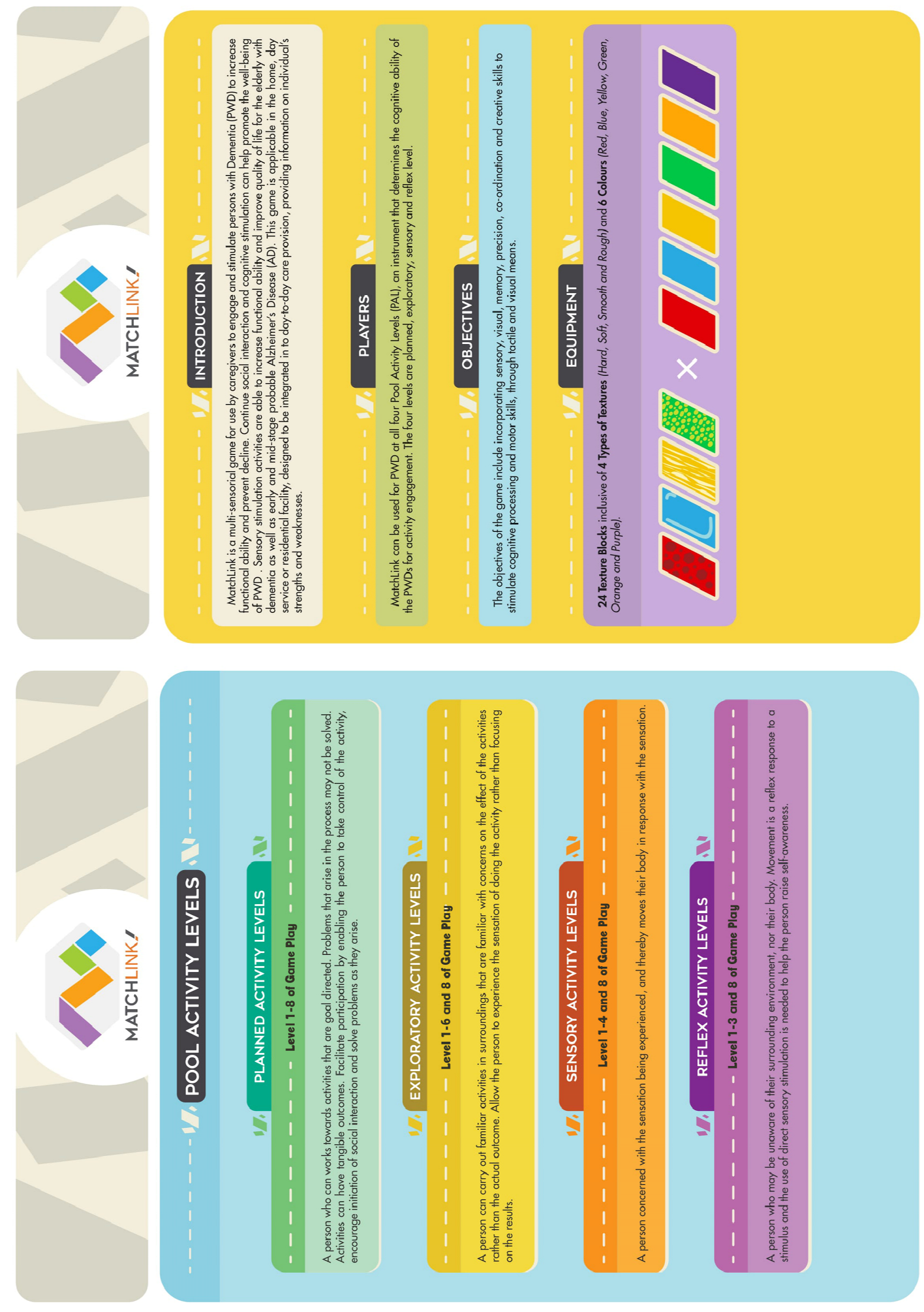

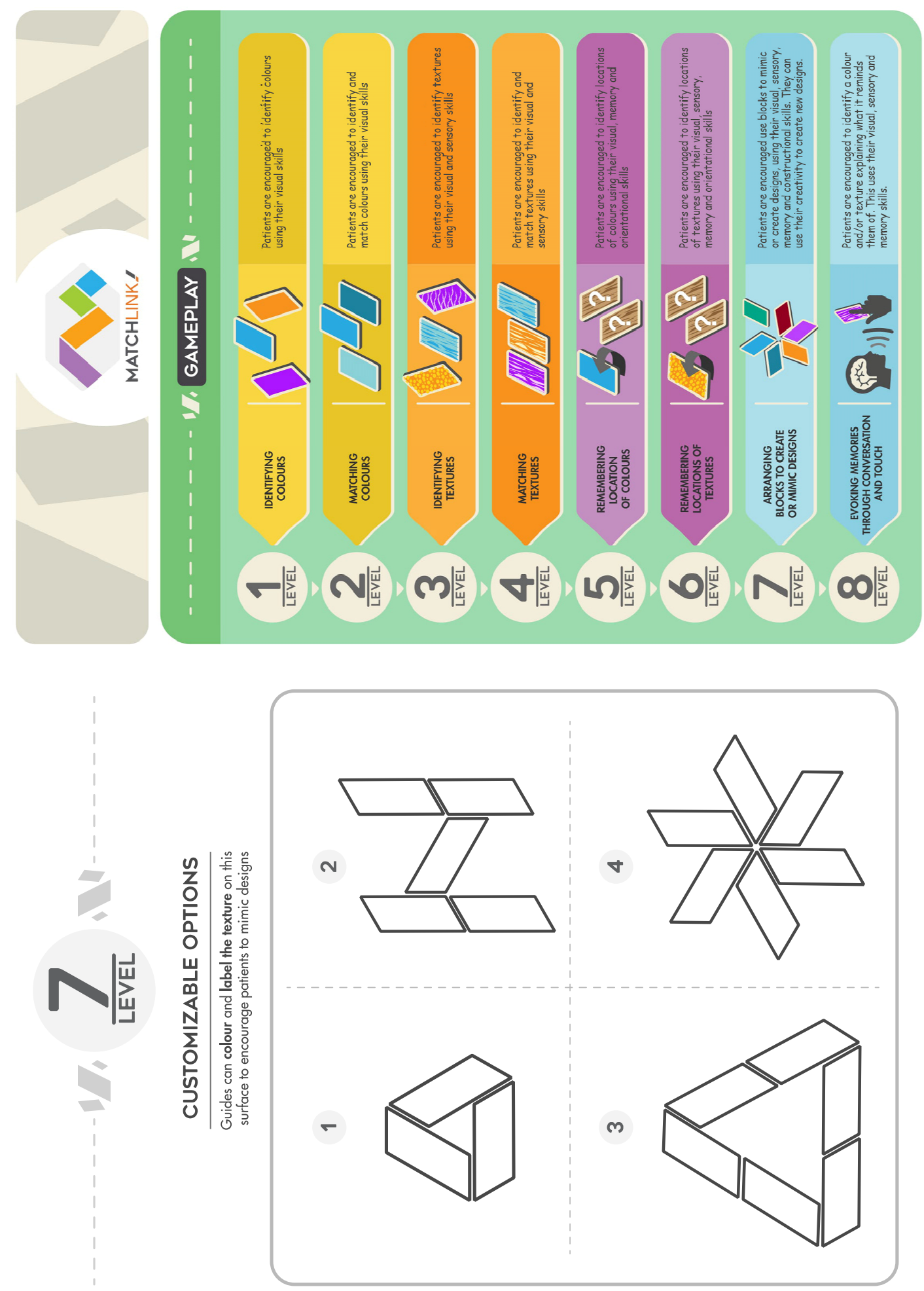

Cassandra Ei Lyn Seah, Product Designer

Nanyang Technological University, School of Art Design and Media (Product Design)

Blk 205, Boon Lay Drive \#05-07, 640205 Singapore, Singapore

Email: cassandra.seah@hotmail.com 